\title{
Detection and Characterization of Prevotella Intermedia and Its In Vitro Susceptibility to Selected Antimicrobial Agents in Chronic Periodontitis and Acute Myocardial Infarction
}

\section{Deepa Anumala, ${ }^{A}$ Mohan Kumar Pasupuleti, ${ }^{B}$ Ravindra Reddy Nagireddyc}

AReader, Department of Periodontics, CKS Theja Dental College, Tirupati, India. BAssociate Professor, Department of Periodontics, Vishnu Dental
College, Bhimavaram, India.

CProfessor and Head, Department of Periodontics, CKS Theja Dental College, Tirupati, India.
Accepted for publication: February 18, 2019

\begin{abstract}
Background: Periodontal disease has been reported to play a causative role in acute myocardial infarction (AMI), which may add to the various risk factors associated with coronary heart disease. The objective of the present study was to investigate the presence of Prevotella intermedia - an established periodontal pathogen - in subgingival plaque samples of chronic periodontitis and AMI patients in order to identify a possible association, and to evaluate the susceptibility of Prevotella intermedia to nine antimicrobial agents. Methods: After undergoing screening for eligibility, a total of 50 subjects were included in the present study. Twenty patients were diagnosed with $A M I$ and generalized chronic periodontitis (Group I), 20 patients were diagnosed with only AMI (Group II), and 10 subjects were healthy controls (Group III). The isolated Prevotella intermedia strains were tested for susceptibility to bacitracin, chloramphenicol, penicillin G, polymyxin, gentamycin, neomycin, tetracycline, cefotaxime, and cefoxitin using an antibiotic zonescale to determine minimum inhibitory concentrations (MICs). Results: Periodontal pathogens were identified by phenotypic and enzymatic methods. The mean bacterial load of Prevotella intermedia species was higher in Group I compared to Group II and Group III. It was also found that pencillin G, gentamycin, neomycin, tetracycline, cefotaxime, and cefoxitin inhibited $90 \%$ of Prevotella intermedia, whereas bacitracin, chloramphenicol, and polymyxin inhibited $80 \%$ of Prevotella intermedia. Thus, only $10 \%$ of Prevotella intermedia were resistant to these antibiotics. Conclusion: The present study confirms that Prevotella intermedia is associated with chronic periodontitis and AMI.
\end{abstract}

Keywords: Acute myocardial infarction; periodontal disease; Prevotella intermedia; antimicrobial susceptibility.

\section{Introduction}

Coronary heart disease results from several environmental and genetic risk factors such as age, abnormal serum lipids, diabetes, smoking, hypertension, and viral and bacterial infections. These known risk factors, independently or combined, are involved in both myocardial infarction and atherosclerosis which are the leading cause of adult mortality and morbidity throughout the world. 1,2,3 A relationship between periodontal disease and systemic diseases such as cardiovascular disease has been proposed, as they share common risk factors including age, abnormal serum lipids, diabetes, smoking, and viral and bacterial infections. Haffajee and Socransky suggested that several subgingival species, including Porphyromonas gingivalis, Prevotella intermedia, and Aggregatibacter actinomycetemcomitans, were associated with atheromatous lesions in the carotid arteries and found that over $40 \%$ of atheromas contain antigens for these periodontal pathogens. The endotoxins released from subgingival species may pass into systemic circulation and contribute to the formation of atheromas. The basis for the 
biological mechanism of this relationship has been acknowledged and has driven an active field of investigation into possible association and causuality. ${ }^{2,3}$

Black pigmented, gram negative anaerobic pathogens such as Prevotella intermedia are involved in both periodontal infections and systemic conditions including myocardial infarction. A variety of microbes with different antimicrobial susceptibility profiles may cause periodontal disease, and as such, selection of antimicrobial agents should be based on proper microbial diagnosis and sensitivity testing while taking into consideration the patient's medical status. Patients who fail to stabilize following mechanical or surgical therapy are often administered antibiotics. ${ }^{4}$ Thus, the aim of the present study was to evaluate the presence of Prevotella intermedia as a possible bacterial species of significance in patients who present with acute myocardial infarction (AMI) and chronic periodontitis, and also to examine the susceptibility of Prevotella intermedia to nine antimicrobial agents.

\section{Materials and Methods}

A total of 50 subjects were included in the present study: 20 were diagnosed with $\mathrm{AMI}$ and chronic generalized periodontitis (Group I), 20 were diagnosed with only AMI (Group II), and 10 healthy controls (Group III). 5 Group I and Group II patients were admitted to the Department of Cardiology, Sri Venkateswara Institute of Medical Sciences (SVIMS). Approval was obtained from the ethical board of SVIMS to conduct the present cross-sectional study. AMI was verified by typical changes on an electrocardiogram and alteration of serum levels of total cholesterol ( $\mathrm{CHO}$ ), high density lipoprotein (HDL), low density lipoprotein (LDL), triglycerides (TG), creatine kinase (CK), and creatine kinase-muscle/brain (CK-MB). All subjects had at least 14 teeth and had not received periodontal therapy or antibiotics six months prior to their recruitment in the present study. Group I patients had probing pocket depths (PPD) greater than $5 \mathrm{~mm}$ and a clinical attachment loss (CAL) greater than $1 \mathrm{~mm}$ in $>30 \%$ of sites. Group II and Group III subjects had probing pocket depths of less than $3 \mathrm{~mm}$ and no CAL.

\section{Oral Examination}

AMI patients were clinically examined three to four days after admission to the cardiology department. A clinical examination was administered which assessed the periodontal status of each patient and included the following parameters: plaque index (PI), gingival index (GI), PPD, Russell's periodontal index, and CAL. ${ }^{6}$

\section{Laboratory Analysis}

Blood samples were withdrawn from all patients upon admission. Serum CHO, HDL, LDL, TG, CK, and $C K-M B$ were all determined by means of an AutoAnalyzer in the clinical laboratory.

\section{Microbiological Tests}

Culture methods for recovery of bacteria are still the gold standard in microbiology. After subgingival plaque samples were collected, they were immediately transferred to a thyoglycolate agar medium (Figure 1). Prevotella intermedia was identified in a pure culture grown in a blood agar anaerobic medium. Gram staining was employed for preliminary identification of Prevotella intermedia and its pleomorphic morphology. As Prevotella intermedia is an aggressive periodontal pathogen, specific pathogenic characteristics were identified including its proteolytic and fibrinolytic activity and the presence of a capsule. Its catalase negative characteristic was also identified.

Figure 1. Plaque sample collected with paper point

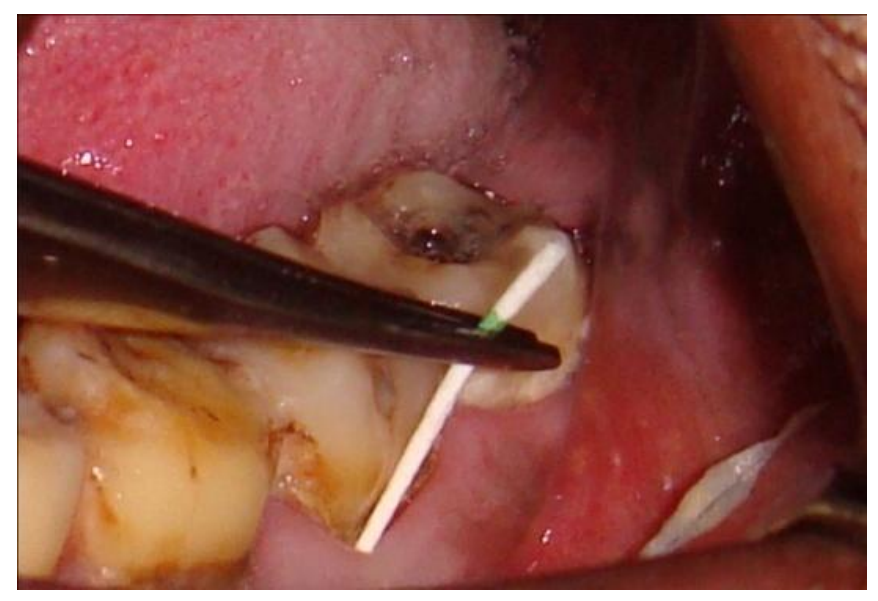

\section{Antimicrobial Susceptibility}

An inoculum of 105 CFU was delivered with a multipoint inoculator to supplemented MuellerHinton agar. The plates were incubated at $37^{\circ} \mathrm{C}$ for 48 hours in jars filled with a gas mixture $\left(85 \% \mathrm{~N}_{2}, 5 \% \mathrm{CO}_{2}, 10 \% \mathrm{H}_{2}\right)$. The isolated Prevotella intermedia strains were tested for susceptibility to bacitracin, chloramphenicol, penicillin $G$, polymyxin, gentamycin, neomycin, tetracycline, cefotaxime, and cefoxitin using a antibiotic zonescalea to determine minimum

a HiMedia® Laboratories Pvt. Ltd. 
inhibitory concentrations (MICs). The antibiotic zonescale was provided in two hexa disks: Hexa Anaerobic-1 and Hexa Universal-1 with antibodies; Hexa Anaerobic-1 contained tetracycline, cefotaxime, and cefoxitin, while Hexa Universal-1 contained bacitracin, chloramphenicol, pencillin $G$, polymyxin, gentamycin and neomycin. The lowest concentration of antimicrobial agents that showed no growth was recorded as the MIC. The susceptibility of Prevotella intermedia was determined by referring to the MICs zone size interpretive standards chart of the National Committee for Clinical Laboratory Standards (NCCLS), which was provided by HiMediaß.

\section{Statistical Analysis}

The means and proportions of the major risk factors and diagnostic markers for AMI, as well as the clinical periodontal parameters were calculated for the three groups and the value of the F-test and mean within group variances was determined by analysis of variance (ANOVA) (Tables 1 and 2). The F-test was used to specify if a group of variables was jointly significant.

\section{$\underline{\text { Results }}$}

Table 1 demonstrates the mean and standard deviation (SD) of the clinical periodontal parameters for all groups. Mean PI, GI, PPD, Russell's index, and CAL were higher in Group I compared to Group II and Group III. The F-value was clinically significant. Table 2 demonstrates the mean and SD of the major risk factors and diagnostic serum enzymes for AMI for all groups. Group I showed higher levels of $\mathrm{CHO}$, LDL, TG, $C K$, and $C K-M B$ compared to Group II and Group III, whereas HDL were lower in Group I compared to Group II and Group III . The Fvalue was clinically significant.

Table 1. Clinical periodontal parameters

\begin{tabular}{|c|c|c|c|c|c|}
\hline & Mean PI & Mean GI & $\begin{array}{c}\text { Mean PPD } \\
(\mathrm{mm})\end{array}$ & $\begin{array}{c}\text { Russell's } \\
\text { Periodontal } \\
\text { Index }\end{array}$ & $\begin{array}{c}\text { Mean CAL } \\
(\mathrm{mm})\end{array}$ \\
\hline Group I & 2.73 & 2.58 & 6.10 & 3.22 & 2.23 \\
\hline Group II & 2.35 & 1.67 & 2.20 & 0.66 & 0 \\
\hline Group III & 1.16 & 1.08 & 1.90 & 0.70 & 0 \\
\hline Significance & $\mathrm{F}=49.249$ & $\mathrm{~F}=58.175$ & $\mathrm{~F}=113.436$ & $\mathrm{~F}=108.915$ & $\mathrm{~F}=52.253$ \\
\hline
\end{tabular}

High clinical periodontal parameters in Group I compared to Group II and Group III. F-value is significant. PI, plaque index; GI, gingival index; PPD, probing pocket depth; and CAL, clinical attachment loss.

Table 2. Lipid profiles and diagnostic serum enzymes for acute myocardial infarction

\begin{tabular}{|c|c|c|c|c|c|c|}
\hline & $\begin{array}{c}\text { Mean CHO } \\
(\mathrm{mg} / \mathrm{dL})\end{array}$ & $\begin{array}{c}\text { Mean HDL } \\
(\mathrm{mg} / \mathrm{dL})\end{array}$ & $\begin{array}{c}\text { Mean LDL } \\
(\mathrm{mg} / \mathrm{dL})\end{array}$ & $\begin{array}{c}\text { Mean TG } \\
(\mathrm{mg} / \mathrm{dL})\end{array}$ & $\begin{array}{c}\text { Mean CK } \\
(\mathrm{U} / \mathrm{L})\end{array}$ & $\begin{array}{c}\text { Mean CK-MB } \\
(\mathrm{U} / \mathrm{L})\end{array}$ \\
\hline Group I & 260.50 & 41.45 & 162.30 & 341.70 & 343.85 & 71.25 \\
\hline Group II & 225.25 & 42.05 & 154.90 & 313.35 & 223.55 & 43.80 \\
\hline Group III & 184.00 & 45.60 & 121.20 & 164.00 & 191.40 & 37.70 \\
\hline Significance & $\mathrm{F}=62.499$ & $\mathrm{~F}=2.322$ & $\mathrm{~F}=57.832$ & $\mathrm{~F}=51.530$ & $\mathrm{~F}=6.981$ & $\mathrm{~F}=57.832$ \\
\hline
\end{tabular}

Low HDL and high LDL, TG, and CHO levels in Group I compared to Group II and Group III. F -value is significant. CHO, total cholesterol; HDL, high density lipoprotein; LDL, low density lipoprotein; TG, triglycerides; CK, creatine kinase; and CK-MB, creatine kinase-muscle/brain. 
Gram staining and bacterial enzymatic reactions demonstrated a higher quantity of Prevotella intermedia in Group I compared to Group II and Group III. Figure 2 demonstrates $50 \%$ positive bacterial enzymatic reactions in Group I, 30\% positive enzymatic reactions in Group II, and $0 \%$ in Group III. Moreover, our results revealed that the total bacterial load and Prevotella intermedia in specific, was significantly higher in Group I compared to Group II and Group III in the following order: Group I > Group II > Group III.
Table 3 shows the susceptibility of Prevotella intermedia to various antibacterials by comparing the recorded MICs to the MICs zone size interpretive standards chart of the National Committee for Clinical Laboratory Standards (NCCLS). It was found that pencillin $G$, gentamycin, neomycin, tetracycline, cefotaxime, and cefoxitin inhibited $90 \%$ of Prevotella intermedia whereas bacitracin, chloramphenicol, and polymyxin inhibited $80 \%$ of Prevotella intermedia. Thus, only $10 \%$ of Prevotella intermedia were resistant to these antibacterial drugs.

Figure 2. Positivity in enzymatic reactions of Prevotella intermedia

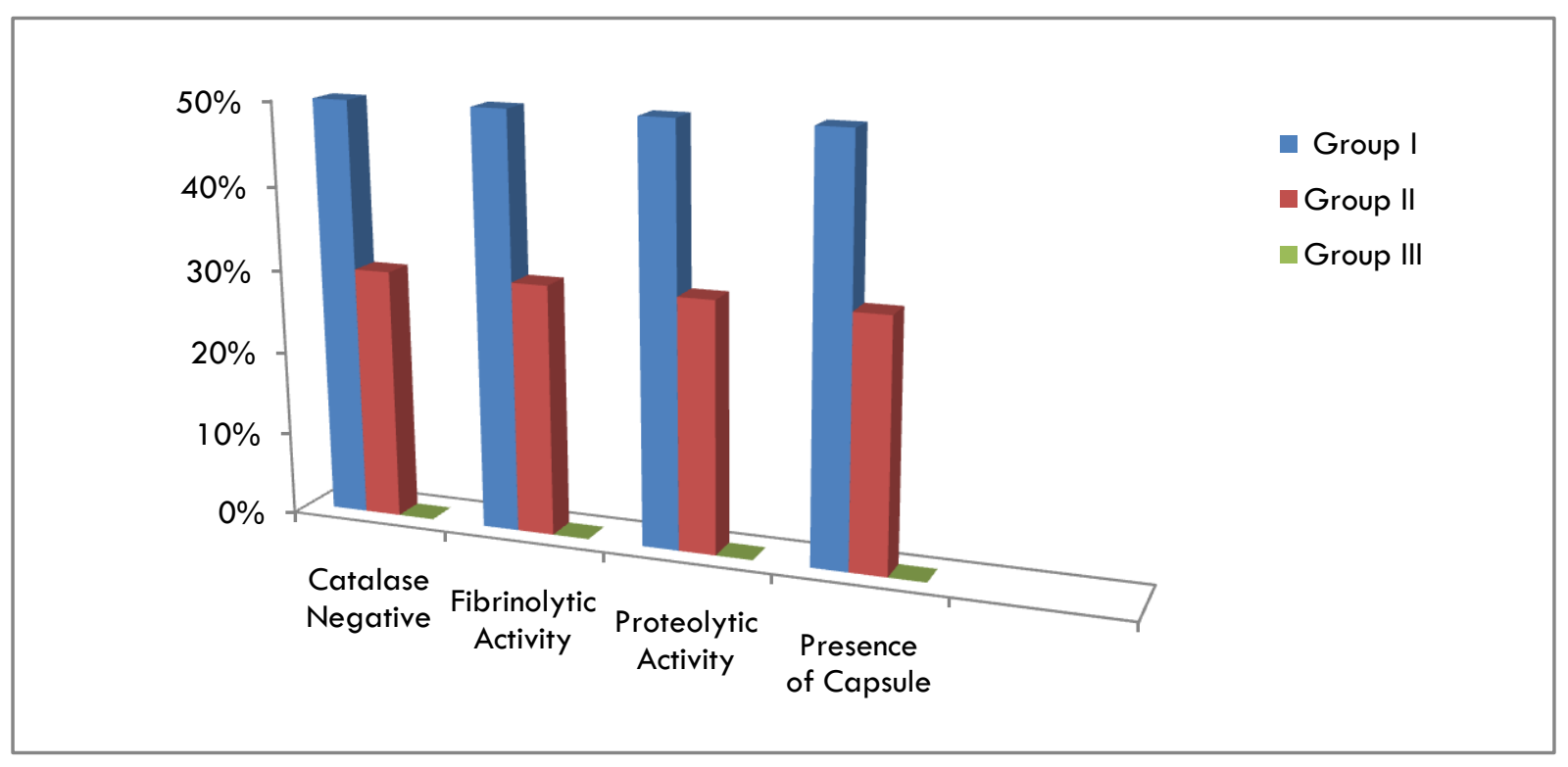

Table 3. Antimicrobial susceptibility of Prevotella intermedia

\begin{tabular}{|c|c|c|c|c|c|c|}
\hline & \multicolumn{3}{|c|}{$\begin{array}{c}\text { Zone Size Interpretative Chart } \\
\text { Provided by HiMedia@ }\end{array}$} & $\begin{array}{c}\text { MICs Of Prevotella intermedia to Antimicrobial } \\
\text { Agents in the Present Study (\%) }\end{array}$ \\
\hline Antibiotic & Resistant & $\begin{array}{c}\text { Intermediate } \\
\text { Sensitive }\end{array}$ & Sensitive & Resistant & $\begin{array}{c}\text { Intermediate } \\
\text { Sensitive }\end{array}$ & Sensitive \\
\hline Bacitracin & $8 \mathrm{~mm}$ & $9-12 \mathrm{~mm}$ & $13 \mathrm{~mm}$ & $20 \%$ & $30 \%$ & $50 \%$ \\
\hline Chloramphenicol & $12 \mathrm{~mm}$ & $13-17 \mathrm{~mm}$ & $18 \mathrm{~mm}$ & $20 \%$ & $20 \%$ & $60 \%$ \\
\hline Pencillin G & $13 \mathrm{~mm}$ & $13-18 \mathrm{~mm}$ & $18 \mathrm{~mm}$ & $10 \%$ & $0 \%$ & $90 \%$ \\
\hline Polymyxin & $11 \mathrm{~mm}$ & $10-12 \mathrm{~mm}$ & $12 \mathrm{~mm}$ & $20 \%$ & $10 \%$ & $70 \%$ \\
\hline Gentamycin & $12 \mathrm{~mm}$ & $13-14 \mathrm{~mm}$ & $15 \mathrm{~mm}$ & $0 \%$ & $20 \%$ & $80 \%$ \\
\hline Neomycin & $14 \mathrm{~mm}$ & $13-16 \mathrm{~mm}$ & $17 \mathrm{~mm}$ & $10 \%$ & $10 \%$ & $80 \%$ \\
\hline Tetracycline & $14 \mathrm{~mm}$ & $15-18 \mathrm{~mm}$ & $19 \mathrm{~mm}$ & $0 \%$ & $20 \%$ & $80 \%$ \\
\hline Cefotaxime & $14 \mathrm{~mm}$ & $15-22 \mathrm{~mm}$ & $23 \mathrm{~mm}$ & $0 \%$ & $20 \%$ & $80 \%$ \\
\hline Cefoxitin & $14 \mathrm{~mm}$ & $15-17 \mathrm{~mm}$ & $18 \mathrm{~mm}$ & $0 \%$ & $10 \%$ & $90 \%$ \\
\hline
\end{tabular}

Pencillin G, gentamycin, neomycin, tetracycline, cefotaxime, and cefoxitin inhibited $90 \%$ of Prevotella intermedia, whereas bacitracin, chloramphenicol, and polymyxin inhibited $80 \%$ of Prevotella intermedia. 


\section{Discussion}

Periodontal diseases are a group of inflammatory conditions of the supporting tissue of the teeth caused by bacteria. It is believed that bacteria associated with periodontal disease enter the bloodstream and travel to major organs such as the heart, thereby contributing to the formation of artery-clogging plaques. ${ }^{5}$ Black-pigmented, gram-negative oral anaerobes such as Prevotella intermedia are regarded as pathogens in chronic periodontitis and are involved in both oral and non-oral infections.

After assessing the periodontal status and lipid profiles in all three groups included in the present study, periodontal parameters and risk factors were found to be higher in Group I compared to Group II and Group III. These results are in agreement with Stein et al. ${ }^{10}$

Nonnenmacher et al. and Mane et al.
found that Prevotella intermedia was a frequently detected microorganism in periodontal disease and was identified by its fibrinolytic and proteolytic activity and by being catalase negative and encapsulated. ${ }^{11,12}$ After assessing the periodontal status and lipid profiles of all three groups included in the present study, we collected subgingival plaque samples in order to test for Prevotella intermedia which was identified by phenotypic characteristics demonstrated through bacterial Gram staining and culture methods. These methods confirmed the presence of Prevotella intermedia in subgingival plaque samples of both chronic periodontitis and $\mathrm{AMI}$ patients.

Patients who do not respond to common mechanical or surgical periodontal therapy are often administered antibiotics as an adjunct to conventional treatment. According to Andrés et al. $14 \%$ of Prevotella intermedia were resistant to $\beta$-lactam antibiotics (pencillins and/or cephalosporins) whereas our study shows that only $10 \%$ were resistant to these antibiotics. ${ }^{4}$

It may be warranted to stabilize the periodontal condition of patients with cardiovascular disease through non-surgical therapy that includes both scaling and root planing (SRP) and adjunctive antimicrobial therapy. The fact that the load of Prevotella intermedia was higher in the chronic periodontitis and $A M I$ group (Group I) compared to the other groups, and that the load was higher in the AMI group (Group II) compared to the healthy control group (Group III), hints to the possibility that this particular pathogen may be linked to the onset of cardiovascular disease.

\section{References}

1. Emingil G, Buduneli E, Aliyev A, Akilli A, Atilla $G$. Association between periodontal disease and acute myocardial infarction. J Periodontal. 2000 Dec; 71(12):1882-86. https://doi.org/10.1902/jop.2000.71.1 2.1882

2. Haffajee $A D$, Socransky SS. Microbial etiological agents of destructive periodontal diseases. Periodontol 2000. 1994 Jun; 5:78-111. https://doi.org/10.1111/i.16000757.1994.tb00020.x

3. Lockhart PB, Bolger AF, Papapanau PN, et al. Periodontal disease and atherosclerotic vascular disease: does the evidence support an independent association?: a scientific statement from the American Heart Association. Circulation. 2012 May 22; 125(20):2520-44.

https://doi.org/10.1161/CIR.0b013e31 $825719 f 3$

4. Andrés MT, Chung WO, Roberts MC, Fiero JF. Antimicrobial Susceptibilities of Porphyromonas gingivalis, Prevotella intermedia, and Prevotella nigrescens spp. Isolated in Spain. Antimicrob Agents Chemother. 1998 Nov.; 42(11):30223023.

5. Rehman MM, Salama RI. Association between periodontal disease and cardiovascular disease. Pak J Med Sci. April-June 2004; 20(2):151-156.

6. Löe H. The Gingival Index, the Plaque Index and the Retention Index Systems. J Periodontol. 1967 Nov-Dec; 38(6): Suppl:610-6.

https://doi.org/10.1902/jop.1967.38.6. 610

7. Armitage GC. Periodontolal diagnoses and classification of periodontal diseases. Periodontol 2000. 2004; 34:921.

8. Haraszthy VI, Zambon JJ, Trevisan $M$, Zeid $M$, Genco RJ. Identification of periodontal pathogens in atheromatous plaques. J Periodontol. 2000 Oct; 71 (10): 1554-60.

https://doi.org/10.1902/jop.2000.71.1 0.1554

9. Genco R, Offenbacher S, Beck J. Periodontal disease and cardiovascular 
disease: epidemiology and possible mechanisms. J Am Dent Assoc. June 2002; 133: Suppl:14S-22S.

10. Stein JM, Kuch B, Conrads G, et al. Clinical periodontal and microbiologic parameters in patients with acute myocardial infarction. I Periodontol. $2009 \quad$ Oct; 80(10):1581-9. https://doi.org/10.1902/jop.2009.090 170

11. Nonnenmacher C, Mutters R, Flores de Jacoby L. Microbiological characteristics of subgingival microbiota in adult periodontitis, localized juvenile periodontitis and rapidly progressive periodontitis subjects. Clin Microbiol Infect. April 2001; 7(4):213-217. https://doi.org/10.1046/i.1469$0691.2001 .00210 . x$

12. Mane AK, Karmarkar AP, Bharadwaj RS. Anaerobic Bacteria in Subjects with
Chronic Periodontitis and in Periodontal Health. J Oral Health Comm Dent. September 2009; 3(3):49-51.

Conflicts of interest: The authors declared no conflicts of interest related to this work.

\section{Corresponding author:}

Dr. Deepa Anumala, MDS

Reader, Department of Periodontics

CKS Theja Institute of Dental Sciences and Research

Chadalawada Nagar, Renigunta Road Tirupati, Andhra Pradesh-517506 India

E-mail: anumala.deepas@gmail.com Phone: +919618501146

This is an open access article distributed under the Creative Commons Attribution-

Noncommercial-NoDerivatives 4.0 International (CC BY-NC-ND 4.0) License. 